\title{
RELAPSING PERICARDITIS
}

Jordi Soler-Soler, Jaume Sagristà-Sauleda, Gaietà Permanyer-Miraldá

Heart 2004;90:1364-1368. doi: 10.1136/hrt.2003.026120

R elapsing or recurrent pericarditis is probably the most troublesome complication of acute pericarditis, and represents one of the greatest therapeutic challenges among the disorders of the pericardium.

\section{DEFINITIONS}

The term relapsing pericarditis includes two clinical types, the incessant type and the intermittent type of recurrent pericarditis. The term incessant applies to those patients in which discontinuation or attempts to wean from anti-inflammatory treatment nearly always ensures a relapse in a period of less than six weeks. ${ }^{1}$ This situation can be seen after discontinuation of the usual anti-inflammatory drugs (aspirin, indomethacin, or ibuprofen) but is particularly frequent and worrying in patients on steroids. Some of these patients may be said to be "steroid hooked" and, in fact, these patients can show a threshold level of prednisone treatment (usually between 10-20 mg) below which relapse is probable. The term "intermittent" refers to those patients with symptom-free intervals conventionally longer than six weeks without treatment.

\section{FREQUENCY}

The possibility of recurrences was already recognised in early descriptions of acute pericarditis. ${ }^{2}$ Their frequency in clinical series including more than 40 patients $^{3-7}$ varies between $8-80 \%$ (average $24 \%$ ), with no distinction between incessant and intermittent types. In our experience, ${ }^{7}$ one or more recurrences were present in 44 of 221 consecutive patients with acute idiopathic pericarditis

\section{AETIOLOGY AND AETIOPATHOGENESIS}

The most typical form of relapsing pericarditis (table 1) occurs after a first episode of idiopathic benign pericarditis, presumably of viral origin. The viruses most frequently implicated are enterovirus, mainly coxsackie B. Higher concentrations of immunoglobulin IgM antibodies have been found in patients with relapses, which can express a persistent viral antigenic stimulation due to persistent viral infection or new exposure to viral illness. Autoimmune responses can certainly play a role in the pathogenesis of recurrent idiopathic or postviral pericarditis. ${ }^{1}$ Inadequate anti-inflammatory treatment of the index attack can explain the relapses in some cases. However, there are no controlled clinical trials about the optimal duration of treatment and the appropriate doses of the drugs. French authors ${ }^{8}{ }^{9}$ have suggested that corticosteroid treatment given in the index attack can favour the occurrence of relapses because of their deleterious effect of viral replication. In the series of Raatikka and colleagues ${ }^{10}$ the mean number of relapses in steroid treated patients was much higher than those not so treated $(8.3 v 4.5)$, during a follow up period of four years. Relapsing pericarditis can also occur in the post-myocardial and postpericardial injury syndromes; relapses after open heart surgery seem to be more frequent in children and adolescents, especially after atrial septal defect closure. ${ }^{10}$ An immunopathologic mechanism is probably the cause of these relapses as anti-heart antibodies have been found in some cases. Genetic factors may play a role in the development of a relapse as well. ${ }^{10}{ }^{11}$ Unlike idiopathic recurrent pericarditis, these cases may eventually evolve to constrictive pericarditis

See end of article for authors' affiliations

Correspondence to: Dr Jordi Soler-Soler, Servei de Cardiología, Hospital Universitari Vall d'Hebron, P. Vall d'Hebron, 119-129, 08035 Barcelona, Spain; jsoler@vhebron.net (this especially occurs in those patients who suffer a tamponade caused by haemopericardium in the index attack). ${ }^{1}$ An immunopathologic mechanism seems to be evident in the relapsing pericarditis of vasculitis-connective tissue disease, especially disseminated lupus erythematosus. Infectious pericarditis caused by specific agents, such as bacterial and tuberculous pericarditis, usually have a subacute or a protracted chronic course with unremitting symptoms, even on common anti-inflammatory drugs, but do not present with a true picture of relapsing pericarditis with intervals of symptom-free periods. ${ }^{12}$ Neoplastic pericarditis can show an oscillating clinical course, and, occasionally, apparently self limited pericarditis with subsequent reappearance of pericardial manifestations. ${ }^{13}$ However, patients with pericardial malignancies, either primary or 
Table 1 Possible causes of relapsing pericarditis

- Idiopathic pericarditis

- Viral pericarditis

- Post-myocardial and pericardial injury syndromes

- post-myocardial infarction syndrome

- post-pericardiotomy syndrome

- post-traumatic pericarditis

- Vasculitis-connective tissue diseases

- Other (very rare)

metastatic, usually have a very poor prognosis, with no propensity for reoccurrence or chronicity to become manifest.

In summary, the most frequent causes of relapsing pericarditis are idiopathic or viral pericarditis and postpericardial injury syndromes. In patients without previous cardiac surgery, and in whom connective tissue disease has been ruled out, relapsing-intermittent pericarditis with symptom-free periods longer than six weeks makes the diagnosis of idiopathic/viral pericarditis practically certain. The pathogenesis of the syndrome can be related to persistent or recurrent viral infection, an immunopathologic mechanism, or an inadequate drug treatment regimen. It has been suggested that the administration of corticosteroids during the target episode can propitiate the appearance of recurrences.

\section{CLINICAL MANIFESTATIONS}

Although the clinical manifestations during recurrences are similar to the first episode of pericarditis, the pattern of relapsing pericarditis is quite characteristic in that the index attack usually is the most severe, while subsequent episodes are milder. In particular, objective manifestations of pericarditis such as pericardial friction rub, electrocardiographic changes, and pericardial effusion are clearly less frequent in subsequent episodes than in the index attack, so in some patients the clinical manifestations in relapses are limited to "pericardial" pain only. For example, in the historical series by Fowler and Harbin ${ }^{14}$ that included 31 patients with recurrent acute pericarditis, all patients had pericardial friction rub and evidence of pericardial effusion or characteristic electrocardiographic changes in the index attack, while these manifestations were entirely absent in subsequent relapses in seven patients. In these patients the evidence of

\section{Aetiology and pathogenesis: key points}

- Relapsing pericarditis occurs in around a quarter of patients after a first episode of acute pericarditis, although the rate of patients with multiple episodes is much lower. Relapses may manifest as an incessant (early reappearance of symptoms every time the patient is weaned from treatment) or as an intermittent type (symptom-free intervals longer than six weeks without treatment)

- Aetiology is diverse, but in patients without previous myocardial infarction or cardiac surgery the vast majority of cases are of viral or immunologic origin. Tuberculous, purulent, or neoplastic pericarditis are not recognised causes of relapsing pericarditis

- The pathogenesis can be related to persistent or recurrent viral infection, an immunopathologic mechanism, or an inadequate therapeutic regimen. Corticosteroid administration in the index attack can play a role in the appearance of the disease pericarditis (apart from pericardial pain) was provided by an increased erythrocyte sedimentation rate, white blood cell count, or fever. This clinical pattern may occasionally result in diagnostic problems in anxious patients reporting chest pain without other features. In one series, ${ }^{10}$ pericardial effusion was present in all 15 patients in the first recurrence, but its frequency decreased progressively with the subsequent relapses; a similar pattern was followed by the ECG changes. If significant pericardial effusion is not present in the index attack, it is very unlikely that it develops in subsequent recurrences. This is also true for clinical tamponade that is rare in recurrent pericarditis.

The number of recurrences and the interval between the episodes vary among patients and are not predictable in individual cases, except during corticosteroid weaning as some patients clearly seem to have a threshold level for recurrences. In our experience with 44 patients with recurrent pericarditis (none had received steroids during the index episode), ${ }^{7} 20$ patients had two episodes, 19 patients had three to five episodes, and five patients had more than five episodes, so although recurrences are relatively common, the number of patients with multiple episodes is much lower.

\section{PROGNOSIS}

In the majority of patients, recurrent attacks of chest pain are the only major disabling feature of the disease. In fact, the hallmark of recurrent pericarditis is the combination of two opposite facts: on the one hand, the excellent life prognosis with exceedingly rare severe complications, and, on the other hand, the severe impairment of the quality of life in some patients caused by incessant recurrences. As previously mentioned, tamponade is very rare during recurrences, especially when not present in the index attack. In the series by Fowler and Harbin ${ }^{14}$ tamponade was present in the initial attack in three patients, but in none of the recurrences, similar to a recent series of 15 young patients..$^{10}$ In the series by Robinson and Bridgen ${ }^{4}$ of 33 patients, two patients developed tamponade. Two patients also developed tamponade in the series by Clémenty and colleagues ${ }^{5}$ involving 20 patients. Although constrictive pericarditis may occur in about $1 \%$ of patients with acute idiopathic pericarditis, ${ }^{15}$ it is not a complication of recurrent pericarditis in spite of numerous episodes..$^{41012}$ Similarly, myocardial disease is not a finding of relapsing pericarditis. In the series by Fowler and colleagues, ${ }^{14}$ no patient developed chronic myocardial disease as documented by cardiac dilation, gallop rhythm or cardiac dysfunction, and no patient had cardiac arrhythmias or conduction disturbances.

In summary, the clinical evolution of relapsing pericarditis is characterised by the recurrence of the episodes of acute pericarditis, which frequently are progressively less severe, without clinical tamponade, and without evolution to constrictive pericarditis. Fortunately, only few cases develop the incessant type of relapsing pericarditis. Furthermore, the recurrences tend to vanish in the long term, although some patients may have recurrences after long symptom-free periods.

\section{MANAGEMENT}

Relapsing pericarditis, with well defined clinical episodes and symptom-free periods longer than six weeks, does not raise special diagnostic difficulties. In patients with no previous cardiac surgery or myocardial infarction, no specific diagnostic test needs to be done. Once the possibility of collagen 
Clinical manifestations and evolution: key points

- Symptoms during recurrences are similar to the index attack, the main manifestation being chest pain of pericardial characteristics

- Usually clinical manifestations are more severe in the index attack, while subsequent episodes are milder. Frequently, chest pain is the only manifestation during relapses

- Tamponade is infrequent during relapses, especially when not present in the index attack. Evolution to pericardial constriction is exceedingly rare

- The number of recurrences and the interval between the episodes varies widely among patients. However, recurrences usually tend to vanish in the long term, although some patients may have recurrences after long symptomfree periods

- Life prognosis is excellent, but some patients have severe quality of life impairment caused by frequent recurrences or iatrogenic side effects

vascular disease has been ruled out, relapsing pericarditis is nearly always of viral/immunologic origin, so additional investigations are irrelevant from a practical point of view.

The two main goals of therapy are the treatment of acute episodes and the prevention of subsequent recurrences.

\section{Treatment of acute episodes}

The treatment of acute pericarditis is to relieve the symptoms and to remove pericardial effusion when tamponade is present. Pain and fever are usually satisfactorily managed by using common analgesic-antipyretic drugs, alone or in combination. On an empirical basis, for patients with no contraindications, the drug of first choice is aspirin, 250$500 \mathrm{mg}$ orally every 4-6 hours. In patients with contraindications or intolerance to aspirin, other types of nonsteroidal anti-inflammatory drugs should be used. Paracetamol (acetaminophen), 500-750 mg every 4-6 hours, can be successfully used in patients with mild to moderate symptoms. In patients with severe symptoms, the most effective drugs are indomethacin (25-50 mg every 6-8 hours) and ibuprofen ( $800 \mathrm{mg}$ every 6-8 hours). For patients with severe refractory symptoms, different combinations of these drugs can be attempted (aspirin plus paracetamol, indomethacin plus paracetamol, ibuprofen plus paracetamol).

Very rarely, symptoms are considered to be refractory and the indication for corticosteroids can arise. Corticosteroids are very effective drugs that quickly control pain and pericardial effusion. However, as previously mentioned, corticosteroid administration may favour the relapses whenever the dose is diminished, leading to recurrent pericarditis. In fact, the most important consideration to prevent both recurrent and incessant pericarditis is to avoid corticosteroid treatment. It is probably much better for the patient to endure the symptoms some additional days than administer corticosteroids prematurely. When corticosteroids have already been given for any reason, the challenge is then to wean the patient off them, especially those who are "steroid-hooked". Prednisone can be quickly reduced from initial high doses ( $1 \mathrm{mg} / \mathrm{kg} /$ day) to the threshold level below which symptoms will probably recur (usually $5-15 \mathrm{mg} /$ day). From this point on, the doses of prednisone should be reduced very slowly in decrements of as little as $1 \mathrm{mg}$. Although there are no controlled prospective studies, and therefore no absolute recommendations can be given, doses can usually be reduced at intervals of between one week and two months, and can be given on alternate days. Even with this approach, the patient must be made aware of the likelihood of many setbacks during the course of the disease. If the syndrome recurs, our practice is to not increase again or reinstitute the administration of prednisone, but to try to control the symptoms with aspirin or other non-steroidal anti-inflammatory drugs following the previously mentioned guidelines.

\section{Prevention of recurrences \\ Colchicine}

In the last few years, colchicine has appeared as a useful drug for preventing recurrences, although the mechanism is not fully understood. On the basis of its reported efficacy in the management of familial Mediterranean fever (a condition associated with polyserositis), Rodriguez de la Serna and colleagues $^{16}$ first suggested in 1987 that colchicine might be useful in preventing recurrences of acute pericarditis. In this pioneering study involving three patients with recurrent pericarditis, treatment with colchicine (1 mg/day) enabled patients to be tapered from their corticosteroids, with no relapses during a follow up period of 15-35 months. In a later open label prospective study, Guindo and colleagues ${ }^{17}$ treated nine patients who had suffered at least three relapses (mean 4.3 episodes per patient) despite treatment with aspirin, indomethacin, prednisone, or a combination. Remarkably, all patients had been receiving prednisone (10-60 mg/day). All nine patients responded favourably to colchicine ( $1 \mathrm{mg} /$ day) and no recurrences were observed in any of the patients during treatment. Steroid treatment could be discontinued in all patients after six weeks. The differences between the symptom-free periods before colchicine administration (mean (SD) interval between pericarditis attacks was 3.3 (4.3) months) and after treatment with colchicine (mean (SD) interval 24.3 (16.1) months) was significant. Moreover, the symptom-free period after treatment with colchicine was more than twice the maximum period before treatment in seven patients. In three patients, colchicine treatment was stopped after symptom-free periods of 26,24 , and 18 months, respectively, and one patient experienced a recurrence six months after colchicine treatment was withdrawn.

While these findings are important, some comment seems appropriate. Firstly, the favourable response in all patients is quite surprising, which raises some doubts about the selection of the patients in this small series. Secondly, the series is not a controlled, blinded, prospective study. In fact, the possibility exists that the prolonged symptom-free periods would have spontaneously occurred as the patients' condition evolved. For example, in the series of Fowler and Harbin, ${ }^{14}$ half of the patients ( 15 of 31 ) were asymptomatic for one year or longer, and 12 were asymptomatic for two years or longer. The symptom-free follow up period was four years for one patient, six years for three, and eight years for two. Similarly, in the series of Raatikka and colleagues, ${ }^{10}$ seven out of 15 patients were symptom-free for more than four years. Adler and colleagues ${ }^{18}$ treated eight patients who had suffered 2-4 recurrences of pericarditis despite treatment with indomethacin, aspirin, or prednisone (in all patients). No recurrences were observed in any patient while on colchicine (mean dosage $1 \mathrm{mg} /$ day), and in four patients an exacerbation occurred when colchicine was stopped after a period of 3-8 months. This experience is very similar to that of Guindo and colleagues. ${ }^{17}$ 
Millaire and colleagues $^{19}$ assessed the usefulness of colchicine in recurrent pericarditis before initiating corticosteroid treatment. Nineteen consecutive patients with recurrent pericarditis (two episodes or more) were included in this prospective, open label study. Colchicine was given at a loading dose of $3 \mathrm{mg}$ and a maintenance dose of $1 \mathrm{mg}$ daily for 1-27 months. No recurrences occurred in 14 out of the 19 patients $(74 \%)$ during a mean (SD) follow up of 37.4 (6.5) months. In four out of the 19 patients $(21 \%)$ five recurrences occurred, which resolved without corticosteroids. Only one patient had several recurrences and required corticosteroids. The authors concluded that colchicine offered a very good benefit/risk ratio in this group of patients with recurrent pericarditis and could be used to avoid the need for corticosteroid treatment.

The largest multicentre study on recurrent pericarditis and colchicine ${ }^{20}$ included 51 patients (33 idiopathic, 18 secondary) who were treated with colchicine and followed up for 6-128 months (mean 36 months). Corticosteroids had been given in 29 of them. Before colchicine treatment the number of recurrences ranged from 2-15 (mean 3.58 \pm 3.64 ) with a mean interval between crisis of 2.0 months (range 0.5-19 months). During a total of 1004 patient-months (mean 12 months per patient) of colchicine treatment (loading dose $0.5-3 \mathrm{mg} / \mathrm{day}$, and maintenance dose $0.5-2 \mathrm{mg} / \mathrm{day})$, only seven of 51 patients $(13.7 \%)$ presented with new recurrences. Colchicine was discontinued in 39 patients, and 14 of them $(35.8 \%)$ experienced relapses. These recurrences were generally minor and were effectively controlled in all patients by the reinstitution of colchicine treatment. During the 2333 patient-months of follow up, 31 patients $(60.7 \%)$ remained recurrence-free. Comparison of the symptom-free periods before and after colchicine treatment yielded significant differences.

In contrast to these globally positive results, Raatikka and colleagues $^{10}$ found colchicine was not effective at all; however, the four colchicine treated patients in this study may have had the severest form of relapsing pericarditis, with $30,13,26$, and 14 relapses, respectively.

In a recent preliminary report, ${ }^{21}$ colchicine was tested as the initial treatment for the first episode of acute pericarditis in order to prevent recurrences. This unblinded, randomised trial involved 60 patients who received either $800 \mathrm{mg} / 6-8$ hours of aspirin for 7-10 days with gradual tapering over 2-3 weeks, or the same aspirin regimen plus colchicine $1 \mathrm{mg} /$ day for at least three months. No recurrences were observed after a mean follow up of 15 months in the colchicine group in contrast to a recurrence rate of $20 \%$ in the aspirin group.

Overall, colchicine appears to be a very good drug in the treatment of recurrent pericarditis. In some patients, symptoms can be completely controlled, and in others colchicine can diminish the severity or the frequency of the relapses, allowing prednisone to be discontinued. A smaller percentage of patients do not respond to treatment. The precise proportion of these responses is not exactly known as appropriately designed placebo controlled prospective studies are lacking. It is unfortunate that such a broad acceptance of this drug has been based on rather weak evidence, but on the basis of the available information we advise the administration of colchicine in patients with two or more relapses, and our impression is that colchicine is useful in more than half of these patients. The use of colchicine in any acute
Management: key points

- Once collagen vascular disease (especially disseminated lupus erythematosus) has been ruled out, complex diagnostic procedures are not justified

- Each episode should be treated with aspirin (250$500 \mathrm{mg}$ orally every 4-6 hours) or other non-steroidal anti-inflammatory drugs (paracetamol $500-750 \mathrm{mg}$ every 4-6 hours, indomethacin $25-50 \mathrm{mg}$ every 6-8 hours), alone or in combination

- Corticosteroids should be avoided. In patients who, for some reason, have already been given corticosteroids, the dosage should be reduced very slowly in decrements as small as $1 \mathrm{mg}$ at intervals of one week to two months

- Colchicine should be administered to patients with two or more relapses. Starting doses of $2-3 \mathrm{mg}$ per day are followed by maintenance doses of 0.5-1 mg per day, which should be given for at least one year after the last episode of pericarditis

- Pericardiectomy is a procedure of doubtful efficacy and should be considered only in very exceptional cases

pericarditis as "primary" prevention of recurrences may represent an important step in the management of acute pericarditis if further controlled trials confirm the initial positive results.

\section{High dose steroidal treatment}

In contrast to the current recommendation to avoid corticosteroids in patients with pericarditis, some authors have advocated the opposite-that is, the administration of prednisone at high doses. The pathophysiological basis for this recommendation is the strong immunosuppressive effect of these high doses (and not of the usual lower doses) that allows the inhibition of immune cytolysis mediated by cytotoxic $\mathrm{T}$ cells, which can play a role in the relapses of pericarditis. Melchior and colleagues ${ }^{22}$ successfully interrupted the relapses in two patients given a pulse therapy of corticosteroids, in which $1 \mathrm{~g}$ of methylprednisolone was given on three consecutive days. Similarly, Marcolongo and colleagues $^{23}$ reported a series of 12 patients with recurrent pericarditis, all of whom had been given corticosteroids at low dose without success. A three months course of treatment with prednisone at immunosuppressive dosage was started ( $1-1.5 \mathrm{mg} / \mathrm{kg}$ body weight per day for four weeks, followed by gradual withdrawal). When prednisone reduction was undertaken, all patients started a five month course of treatment with aspirin ( $1.6 \mathrm{~g} /$ day until steroid was stopped, then reduced to $0.8 \mathrm{~g} /$ day). Immunosuppressive treatment resulted in stable remission in all except one patient, who experienced one relapse. Three patients had severe steroid related adverse effects, requiring replacement of prednisone with azathioprine and cyclophosphamide, with a good favourable clinical outcome.

These forms of treatment widen the therapeutic possibilities of recurrent pericarditis, but these experiences have not been validated by other groups, and the risk of side effects from these high doses of corticoids is a matter of concern. The usually self limited course of relapsing pericarditis should caution against the indiscriminate use of such an approach. Again, the lack of proper clinical trials is to be deplored.

\section{Restriction of physical activity}

The role of physical activity in the recurrence and exacerbation of pericarditis is unknown. However, in clinical practice 
it is not uncommon that some patients report a worsening of symptoms apparently provoked by exercise. This is especially frequent in patients with persistent precordial pain as the only manifestation of their illness, while the role of exercise as a trigger of evident inflammatory relapses (fever, pericardial friction rub, pericardial effusion) is much less clear. In any case, it seems reasonable to restrict physical activity, particularly when the patient is weaned from prednisone or other anti-inflammatory drugs. The desirable amount of restriction cannot be known in the absence of appropriately designed controlled trials, but it is advisable to restrict exertion beyond what is necessary to perform domestic tasks and undertake sedentary work.

\section{Pericardiectomy}

Pericardiectomy has been proposed for the relief of refractory relapsing pericarditis, but performing such a procedure does not always result in the end of the recurrences. For example, in the series by Fowler and Harbin, ${ }^{14}$ pericardiectomy was done in nine patients, but clear-cut relief was observed in only two of them. Similarly, in our series of 44 patients, pericardiectomy was undertaken in only two of them, and both patients continued to experience recurrent episodes after their operations. ${ }^{7}$ Thus, pericardiectomy should be considered to be the last resort in patients with severe relapsing pericarditis in whom an adequate drug treatment has failed. Perhaps only the patient who presents with recurrent severe pericardial effusion constitutes the clearest indication for pericardiectomy, but this type of patient is exceedingly rare.

In summary, the aetiologic diagnosis is usually unchallenging, as the vast majority of cases are of viral or immunologic origin. Perhaps the most effective way to prevent relapsing pericarditis is to avoid the use of corticosteroids in the index attack and to manage each episode with aspirin or other nonsteroidal anti-inflammatory drugs. Colchicine can offer a valuable aid, and its administration can be advised in patients with two or more relapses. The possible role of other kinds of treatment (especially immunosuppressive therapy) has not been adequately assessed. Pericardiectomy should be considered only in very exceptional cases.

\section{Authors' affiliations}

J Soler-Soler, J Sagristà-Sauleda, G Permanyer-Miralda, Servei de

Cardiología, Hospital Universitari Vall d'Hebron, Barcelona, Spain

\section{REFERENCES}

1 Spodick DH. Recurrent and incessant pericarditis. In: Spodick DH, ed. The pericardium. A comprehensive textbook. New York: Marcel Dekker, Inc, 1997.

- This chapter constitutes an excellent review on this topic. The aetiologic and pathogenic aspects are well explained, and key points of management are also well discussed.
2 Barnes AR, Burchell HB. Acute pericarditis simulating acute coronary occlusion. A report of 14 cases. Am Heart J 1942;23:247-68.

3 Carmichael DB, Sprague HB, Wyman SM, et al. Acute non-specific pericarditis. Clinical laboratory and follow-up considerations. Circulation $1951 ; 3: 321-31$

4 Robinson J, Bridgen W. Recurrent pericarditis. BMJ 1968;2:272-5.

5 Clémenty J, Jambert H, Dallacchio M. Les pericardites aigües recidivantes: 20 observations. Arch Mal Coeur 1979;72:857-61.

6 Admetlla M, Cequier A, Amer R, et al. Acute idiopathic pericarditis: clinical features, development and complications. Prospective study of 101 cases. Med Clin (Barc) 1985;85:563-7

7 Soler-Soler J, Permanyer-Miralda G, Sagristà-Sauleda J. Pericardial disease. In: New insights and old dilemmas. Dordrecht: Kluwer Academic Publishers, 1990.

- This book provides relevant information about the frequency, number of relapses, aetiology, and outcome in a consecutive series of 221 patients with acute idiopathic pericarditis.

8 Froment R, Perrin A, Saint-Pierre A, et al. Efflorescence de péricardites idiopathiques récidivantes. Rôle probable des antibiotiques et de la corticotherapie. J Med Lyon 1970;25:113-23.

9 Godeau $P$, Derrida JP, Bletry $G$, et al. Péricarditis aiguës récidivantes et cortico-dépendance. A propos de 10 observations. Sem Hop Paris 1975;51:2393-400.

10 Raatikka M, Pelkonen PM, Karjalainen J, et al. Recurrent pericarditis in children and adolescents. J Am Coll Cardiol 2003;42:759-64.

- This is a good report on 15 children and adolescents (seven of them with an operated atrial septal defect) that presented 2-30 recurrences (mean 9.9), with a long term follow up (mean 8 years). Several modalities of treatment are discussed, but none of them seems to have influenced the benign outcome of this syndrome.

11 Deline JM, Cable DG. Clustering of recurrent pericarditis with effusion and constriction in a family. Mayo Clin Proc 2002;77:39-43

12 Sagristà-Sauleda J, Permanyer-Miralda G, Soler-Soler J. Tuberculous pericarditis: ten-year experience with a prospective protocol for diagnosis and treatment. J Am Coll Cardiol 1988;11:724-8

13 Galve E, Permanyer-Miralda G, Tornos $P$, et al. Self-limited acute pericarditis as initial manifestation of primary cardiac tumor. Am Heart $J$ 1992;123:1690-2.

14 Fowler NO, Harbin D. Recurrent acute pericarditis: follow-up study of 31 patients. J Am Coll Cardiol 1986;7:300-5.

- This is an outstanding article that provides very useful information about the natural history and clinical evolution of 31 adults with recurrent pericarditis, with an observation period of 2-19 years. In addition, the efficacy and limitations of different treatments are discussed, including pericardiectomy.

15 Permanyer Miralda G, Sagristà-Sauleda J, Soler-Soler J. Primary acute pericardial disease: a prospective series of 231 consecutive patients. Am J Cardiol 1985;56:623-30.

16 Rodriguez de la Serna A, Guindo J, Marti V, et al. Colchicine for recurrent pericarditis [letter]. Lancet 1987; ii: 1517.

- The first report of the possible therapeutic value of colchicine in three patients with recurrent pericarditis.

17 Guindo J, Rodriguez de la Serna A, Ramió J, et al. Recurrent pericarditis. Relief with colchicine. Circulation 1990;82:1117-20.

- This article is the first report of the efficacy of colchicine in a series of nine patients with recurrent pericarditis.

18 Adler Y, Zandman-Goddard G, Ravid M, et al. Usefulness of colchicine in preventing recurrences of pericarditis. Am J Cardiol 1994;73:916-7.

19 Millaire A, de Groote P, Decoulx E, et al. Treatment of recurrent pericarditis with colchicine. Eur Heart J 1994; 15:120-4.

20 Adler Y, Finkelstein Y, Guindo J, et al. Colchicine treatment for recurrent pericarditis. A decade of experience. Circulation 1998;97:2183-5.

- Review of the available information about colchicine and report of the data of the largest multicentre study on recurrent pericarditis with this drug.

21 Imazio $M$, Demichelis B, Cecchi $E$, et al. Is colchicine the initial mode of treatment for acute pericarditis? Eur Heart J 2003;24(abstract suppl):250.

22 Melchior TM, Ringsdal V, Hildebrandt $\mathrm{P}$, et al. Recurrent acute idiopathic pericarditis treated with intravenous methylprednisolone given as pulse therapy. Am Heart J 1992; 123:1086-8.

23 Marcolongo R, Russo R, Laveder F, et al. Immunosuppressive therapy prevents recurrent pericarditis. J Am Coll Cardiol 1995;26:1276-9. 